\title{
Pacific
}

Journal of

Mathematics

3-VALENT GRAPHS AND THE KAUFFMAN BRACKET

Gregor Masbaum and Pierre Vogel 


\section{3-VALENT GRAPHS AND THE KAUFFMAN BRACKET}

\section{G. Masbaum and P. Vogel}

We explicitly determine the tetrahedron coefficient for the onevariable Kauffman bracket, using only Wenzl's recursion formula for the Jones idempotents (or augmentation idempotents) of the Temperley-Lieb algebra.

1. Statement of the main result. In this paper, we consider unoriented knot or tangle diagrams in the plane, up to regular isotopy. Furthermore, we impose the Kauffman relations [Ka1]

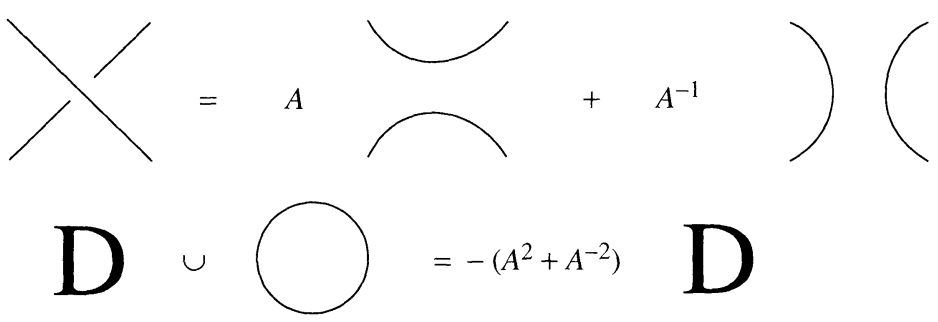

(the first relation refers to three diagrams identical except where shown, and in the second relation, $\mathbf{D}$ represents any knot or tangle diagram). Coefficients will always be in $\mathbf{Q}(A)$, the field generated by the indeterminate $A$ over the rational numbers. With these relations, $(n, n)$ tangles (i.e. tangles in the square with $n$ boundary points on the upper edge and $n$ on the lower edge) generate a finite-dimensional associative algebra $T_{n}$ over $\mathbf{Q}(A)$, called the Temperley-Lieb algebra on $n$ strings (see [Li1] for more details on what follows). Multiplication in $T_{n}$ is induced by placing one diagram above another, and the identity element $1_{n}$ is given by the identity $(n, n)$-tangle. Up to isotopy, there is a finite number of $(n, n)$-tangles without crossings and without closed loops; they form the standard basis of the algebra $T_{n}$. Let $\varepsilon: T_{n} \rightarrow \mathbf{Q}(A)$ denote the associated augmentation homomorphism, i.e. $\varepsilon\left(1_{n}\right)=1$, and $\varepsilon$ is zero on the other basis elements. The algebra $T_{n}$ (with coefficients in $\mathbf{Q}(A)$ ) is semisimple, and in particular there is an augmentation idempotent $f_{n} \in T_{n}$ with the property

$$
f_{n} x=x f_{n}=\varepsilon(x) f_{n}
$$


for all $x \in T_{n}$, and $f_{n}^{2}=f_{n} \cdot{ }^{1}$ These idempotents were first discovered by Jones [J1]. Up to normalisation, they are equal to the "magic weaving elements" of [Ka2]. There is a recursion formula for these idempotents due to Wenzl [We], which we will now state graphically.

We make the convention that writing $n$ beneath a component of a knot or tangle diagram means that this component has to be replaced by $n$ parallel ones ( $n$ may be zero). ${ }^{2}$ We will often say that this component is colored by $n$ (a color is just a non-negative integer). For graphical reasons, we make the further convention that we may omit indicating the color if it can easily be deduced from the rest of the diagram. Let us denote the augmentation idempotent $f_{n} \in T_{n}$ by a little box. Then Wenzl's formula can be stated graphically as follows:

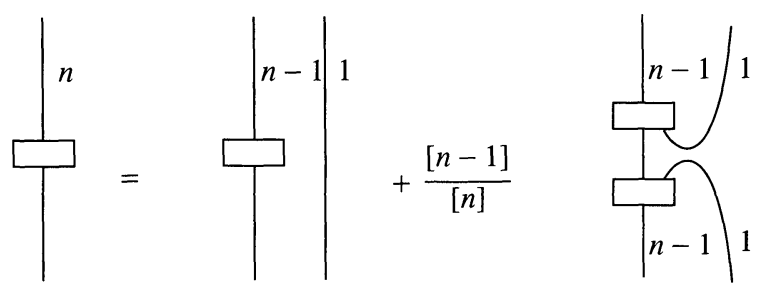

Here, we use the notation $[n]=\left(A^{2 n}-A^{-2 n}\right) /\left(A^{2}-A^{-2}\right)$. For a proof of this formula, see [We] or [Li1]. (Notice that in the last diagram of the formula above, we did not indicate the color of the line joining the two boxes. As explained above, this does not mean that the color is one, but that the color must be calculated form the rest of the diagram. Here clearly the color is $n-2$, because the little boxes represent elements of the Temperely-Lieb algebra on $n-1$ strings.)

Following Kauffman [Ka2], we define a 3-valent vertex as follows. A triple $(a, b, c)$ of colors is called admissible if $a+b+c$ is even and $|a-b| \leq c \leq a+b$ (triangle inequality). Given an admissible triple, we define a 3 -valent vertex by
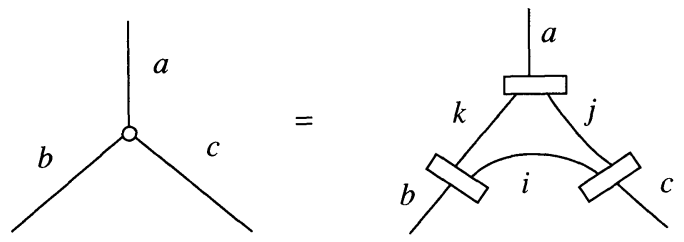

\footnotetext{
${ }^{1}$ In [Li1, Li2], our $f_{n}$ is denoted $f_{n-1}=f^{(n)}$.

${ }^{2}$ This convention is consistent with [Li1, Li2], but not with the graphical conventions of [BHMV1].
} 
The numbers $i=(b+c-a) / 2, j=(a+c-b) / 2, k=(a+b-c) / 2$ of internal lines of the 3-valent vertex will be called its internal colors. Notice that a triple $(a, b, c)$ is admissible if and only if one can find corresponding internal colors.

The goal of this paper is to give an explicit determination of the following three coefficients, using only Wenzl's formula for the augmentation idempotents $f_{n}$.

trihedron coefficient:

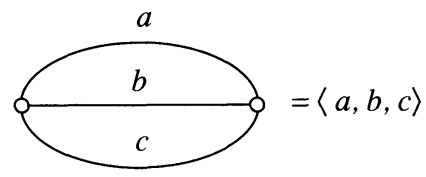

tetrahedron coefficient:

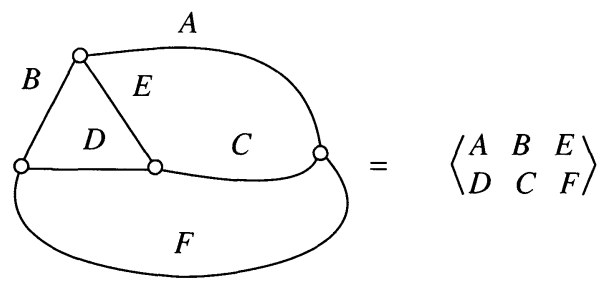

(Of course, the trihedron coefficient is a special case of the tetrahedron coefficient, e.g. $\left\langle\begin{array}{lll}a & a & 0 \\ b & b & c\end{array}\right\rangle=\langle a, b, c\rangle$.)

half-twist coefficient:

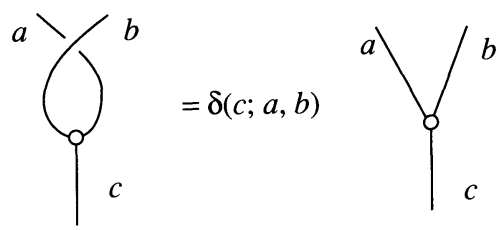

REMARKs. (1) The names trihedron and tetrahedron coefficient are motivated by the fact that one may imagine the corresponding graphs as a regular trihedron or tetrahedron drawn on a sphere. 
(2) Knowing the tetrahedron coefficient is equivalent to knowing the (more popular) recoupling coefficients or 6-j-symbols (see $\S 2$ ).

(3) Reversing the sign of the crossing in the half-twist coefficient replaces $\delta(c ; a, b)$ by its conjugate $\overline{\delta(c ; a, b)}$, where the conjugation on $\mathbf{Q}(A)$ is defined by $\bar{A}=A^{-1}$.

Recall $[n]=\left(A^{2 n}-A^{-2 n}\right) /\left(A^{2}-A^{-2}\right)$. This notation is motivated by the fact that $\left.[n]\right|_{A=1}=n$. Notice $[0]=0$. We also define $[n] !=$ $[1] \cdots[n]$ for $n \geq 1$, and [0]! $=1$.

THEOREM 1 (Trihedron coefficient). Let $(a, b, c)$ be admissible and let $i, j, k$ be the internal colors of a 3-valent vertex $(a, b, c)$. Then

$$
\langle a, b, c\rangle=(-1)^{i+j+k} \frac{[i+j+k+1] ![i] ![j] ![k] !}{[i+j] ![j+k] ![i+k] !} .
$$

THEOREM 2 (Tetrahedron coefficient). Let $A, B, C, D, E, F$ be colors such that the triples $(A, B, E),(B, D, F),(E, D, C)$ and $(A, C, F)$ are admissible. Set $\Sigma=A+B+C+D+E+F$ and

$$
\begin{array}{ll}
a_{1}=(A+B+E) / 2, & b_{1}=(\Sigma-A-D) / 2, \\
a_{2}=(B+D+F) / 2, & b_{2}=(\Sigma-E-F) / 2, \\
a_{3}=(C+D+E) / 2, & b_{3}=(\Sigma-B-C) / 2 . \\
a_{4}=(A+C+F) / 2, &
\end{array}
$$

Then

$$
\left\langle\begin{array}{ccc}
A & B & E \\
D & C & F
\end{array}\right\rangle=\frac{\prod_{i=1}^{3} \prod_{j=1}^{4}\left[b_{i}-a_{j}\right] !}{[A] ![B] ![C] ![D] ![E] ![F] !}\left(\begin{array}{cccc}
a_{1} & a_{2} & a_{3} & a_{4} \\
b_{1} & b_{2} & b_{3}
\end{array}\right)
$$

where

$$
\left(\begin{array}{cccc}
a_{1} & a_{2} & a_{3} & a_{4} \\
b_{1} & b_{2} & b_{3}
\end{array}\right)=\sum_{\max \left(a_{j}\right) \leq \zeta \leq \min \left(b_{\imath}\right)} \frac{(-1)^{\zeta}[\zeta+1] !}{\prod_{i=1}^{3}\left[b_{i}-\zeta\right] ! \prod_{j=1}^{4}\left[\zeta-a_{j}\right] !} .
$$

(Notice that the $a_{j}$ correspond to the four vertices of the tetrahedron, whereas the $b_{j}$ correspond to the three pairs of opposite edges. Moreover, it is easy to verify that the 12 numbers $b_{i}-a_{j}$ are the internal colors of the four 3 -valent vertices.)

THEOREM 3 (Half-twist coefficient). Let $(a, b, c)$ be admissible and let $i=(b+c-a) / 2, j=(a+c-b) / 2, k=(a+b-c) / 2$ be the 
interval colors of $a$ 3-valent vertex $(a, b, c)$. Then

$$
\delta(c ; a, b)=(-1)^{k} A^{i j-k(i+j+k+2)} .
$$

REMARKS. (1) Similar (but not identical) formulas were deduced by Reshetikhin and Kirillov [KR] from the representation theory of the quantum group $U_{q} \mathrm{Sl}_{2} \cdot{ }^{3}$ They are in fact $q$-analogues of formulas that have been used by physicists for a long time in the quantum theory of angular momentum (see for example [BL]). Piunikhin [P] has worked out the precise relationship between Kauffman's definition of a 3-valent vertex, which is the one we use here (up to normalisation), and the definition coming form the representation theory of $U_{q} \mathrm{Sl}_{2}$ (we will state his formula in an appendix to §2). Using the result of [KR], he thus obtains a formula for the tetrahedron coefficient. Nevertheless, it seems fair to say that the approach we take here is a much simpler way to get our formulas, because the representation theory approach involves much more complicated calculations than our approach through the Kauffman bracket.

(2) The results of this paper are used in [BHMV3], where it is shown how the invariants $\theta_{p}$ of [BHMV1, BHMV2] lead to an elementary construction of a "Topological Quantum Field Theory".

The remainder of this paper is organized as follows. In $\S 2$, we will show how the knowledge of the three coefficients above allows one to evaluate any colored link diagram or any colored 3-valent graph. (This is a fact well known to specialists.) The bulk of this paper is $\S 3$, where we will give the proof of Theorems 1-3.

The authors wish to acknowledge helpful conversations with $\mathrm{O}$. Viro, who pointed out a great simplification in our original proof of Theorem 3. The proof of Theorem 3 given here is due to him.

2. Evaluating colored link diagrams and colored 3-valent graphs. This section presents a graphical calculus which allows one to evaluate any colored link diagram or any colored 3-valent graph, using the coefficient determined in Theorems 1-3.

We first need to recall some concepts. Let $\mathscr{B}$ be the $\mathbf{Q}(A)$-module generated by link diagrams in an annulus, modulo regular isotopy and the Kauffman relations. ${ }^{4}$ Given a $k$-component link diagram $L$, and

\footnotetext{
${ }^{3}$ In order to make contact with $q$-formulas and $U_{q} \mathrm{Sl}_{2}$, the correct substitution seems to be $A=-q^{-1 / 4}$. Note that the Kauffman bracket is related to Jones' original $V$-polynomial [J2] through a similar substitution (see [Ka1]).

${ }^{4} \mathscr{B}$ was called $\mathscr{A}$ in [Li2]. It is also the same as the Jones-Kauffman module of a solid torus as defined in [BHMV1], because of the usual correspondence between planar link diagrams up to regular isotopy, and banded links in 3-space.
} 
$b_{1}, \ldots, b_{k} \in \mathscr{B}$, let $\left\langle b_{1}, \ldots, b_{k}\right\rangle_{L}$ denote the Kauffman bracket of the linear combination of link diagrams obtained from $L$ by replacing the $j$ th component by $b_{j}$. Notice that $\langle, \ldots,\rangle_{L}$ is a $k$-linear form on $\mathscr{B} .{ }^{5}$ There is a "trace map" $T_{n} \rightarrow \mathscr{B}$ given by mapping a tangle in the square to the diagram in the annulus obtained by identifying the upper and lower edges of the square. The image of an element $x \in T_{n}$ under this map is called the closure of $x$, and will be denoted by $\hat{x}$. Set

$$
e_{n}=\hat{f}_{n} \in \mathscr{B} \text {. }
$$

It turns out that the $e_{n} \quad(n \geq 0)$ form a basis of $\mathscr{B}$, and hence the meta-bracket of $L$ is determined by the values

$$
\left\langle e_{i_{1}}, \ldots, e_{i_{k}}\right\rangle_{L} \text {. }
$$

We call this the colored bracket polynomial of $L$. This is up to normalisation the same as the colored Jones polynomial appearing in the construction of 3-manifold invariants in $[\mathbf{R T}]$ and $[\mathbf{K M}]^{6}$ (see also [MS]).

The goal of this section is to evaluate $\left\langle e_{i_{1}}, \ldots, e_{i_{k}}\right\rangle_{L}$. With our graphical conventions, this is the value of the diagram obtained form $L$ by writing $i_{j}$ beneath the $j$ th component, and inserting one little box into each component. Such a diagram will be called a colored link diagram. (We may place the little box wherever we want on the component. Also, we may insert several boxes since a box represents an idempotent.) Let $C$ denote the diagram of a circle, and set

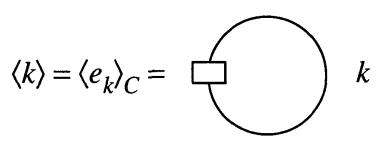

(This was denoted by $\left\langle e_{k}\right\rangle$ in [BHMV1], and is equal to $(-1)^{k}[k+1]=$ $(-1)^{k}\left(A^{2 k+2}-A^{-2 k-2}\right) /\left(A^{2}-A^{-2}\right)$.) Gluing two annuli together so as to get a third endows $\mathscr{B}$ with a multiplication for which $e_{0}=1$ and $e_{1} e_{i}=e_{i+1}+e_{i-1}$ [BHMV1]. It follows easily that in $\mathscr{B}$, one has the equation

$$
e_{i} e_{j}=\sum_{k} e_{k}
$$

(the sum being over all $k$ such that the triple $(i, j, k)$ is admissible).

\footnotetext{
${ }^{5}$ This $k$-linear form was first considered in [Li2]. We call it the meta-bracket of $L$ [BHMV1].

${ }^{6} \mathrm{See}$ also a remark in the proof of Prop. 2.2 in [BHMV2].
} 
This implies the following graphical equation in $T_{i+j}$ :

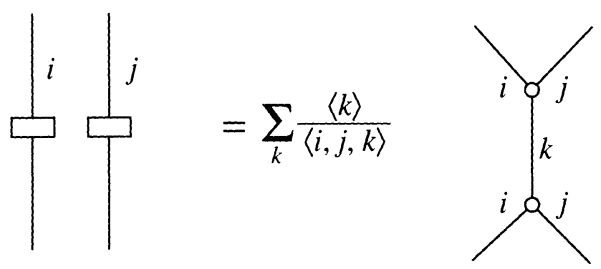

We now show how to use this and the coefficients determined in Theorems $1-3$ to evaluate colored link diagrams. (The rangle of summation is not indicated explicitly in the formulas below. It is determined precisely by the requirement that the colors meeting at a 3-valent vertex should form an admissible triple.) First of all, we can get rid of crossings using the above formula and the half-twist coefficient, as follows:

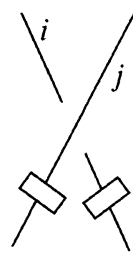

$$
=\sum_{k} \frac{\langle k\rangle}{\langle i, j, k\rangle}
$$
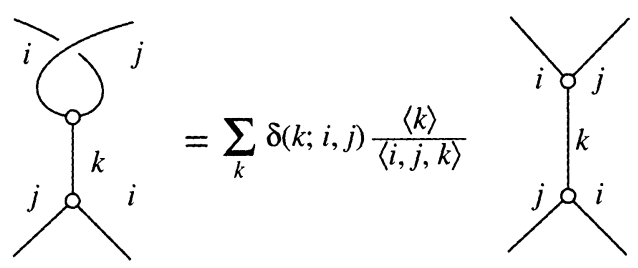

Thus we are left with a colored 3-valent graph. Notice that an edge colored with zero may just as well be removed, since $f_{0}$ is represented by the empty $(0,0)$-tangle. Since

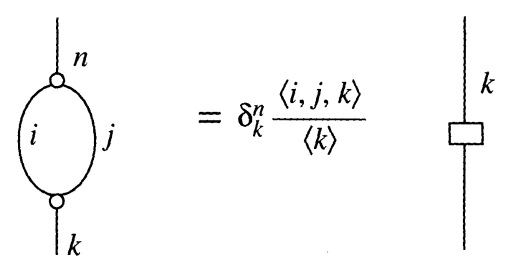

any diangular face (that is a face with precisely two vertices) of a graph can be eliminated. Similarly, any triangular face can be simplified using the tetrahedron coefficient as follows:

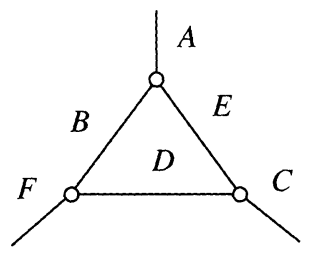

$$
=\frac{\left\langle\begin{array}{lll}
A & B & E \\
D & C & F
\end{array}\right\rangle}{\langle A, F, C\rangle}
$$

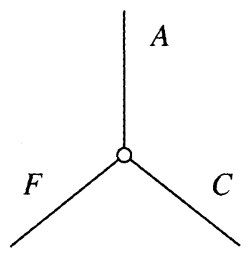


More generally, any triangle, square, pentagon, etc. can be reduced to a diangular face (which can then be eliminated by formula $(*)$ above) using

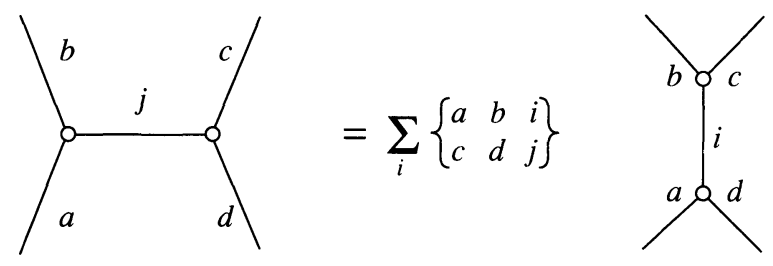

where $\left\{\begin{array}{lll}a & b & i \\ c & d & j\end{array}\right\}$ is the recoupling coefficient or 6-j-symbol defined by

$$
\left\{\begin{array}{lll}
a & b & i \\
c & d & j
\end{array}\right\}=\frac{\langle i\rangle\left\langle\begin{array}{lll}
i & b & c \\
j & d & a
\end{array}\right\rangle}{\langle i, a, d\rangle\langle i, b, c\rangle}
$$

(see [Ka2] for a graphical proof of this formula). We now are left with a connected graph each of whose faces has at most one vertex. As explained above, we may assume no edge is colored by zero. Then if the graph has a vertex, the value is zero, because for $k \geq 1$ one has

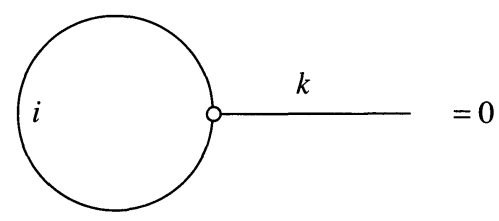

as follows immediately from the fact that $f_{k}$ is the augmentation idempotent. If however the graph has no vertex, then it is simply a disjoint union of circles colored by $k_{i}$ 's, and its value is the product of the $\left\langle k_{i}\right\rangle$ 's. (By the very definition of the Kauffman bracket, the value of a disjoint union of diagrams is equal to the product of the values of the individual components.)

This completes the evaluation of colored link diagrams.

REMARK. The reader will find two more useful graphical identities in the proof of Theorem 3 .

REMARK. In this paper, we have made the convention that writing $n$ beneath a component of a knot or tangle diagram just means that this component has to be replaced by $n$ parallel ones. It is sometimes useful to make the additional convention that writing $n$ beneath a 
component also includes insertion of the idempotent $f_{n}$. For example, this would be convenient when using the graphical calculus to evaluate the colored bracket polynomial of a link diagram. Indeed, in this context it is sufficient to consider only diagrams where each component contains an $f_{n}$, and it would then no longer be necessary to draw the little boxes explicitly. However, for the purpose of this paper, which is to give an elementary proof of Theorems $1-3$, it is useful not to make this additional convention.

Appendix. Comparison with the representation theoretical approach. Here is the relationship of our graphical calculus with the one of [KR] (see [P] for more details). Let $V_{n}$ denote the quantum deformation of the standard irreducible representation of $\mathrm{sl}_{2}$ of spin $n / 2$ (i.e. of dimension $n+1)$. The 3-valent vertices of [KR] represent certain intertwining homomorphisms $K_{a b}^{c}: V_{a} \otimes V_{b} \rightarrow V_{c}, K_{c}^{a b}: V_{c} \rightarrow V_{a} \otimes V_{b}$ as follows:
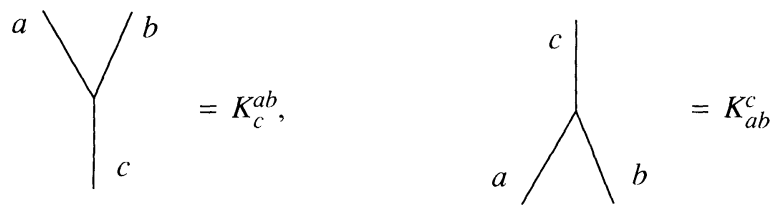

As explained in $[\mathbf{P}]$, the trivalent vertex as defined in $\S 1$ may also be interpreted as an intertwiner, and there are coefficients $\phi_{c}^{a b}, \phi_{a b}^{c}$ such that
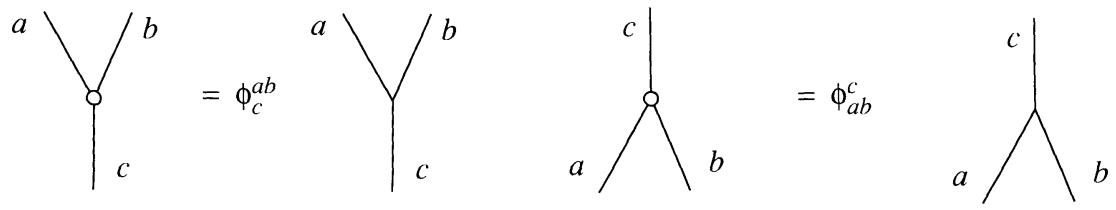

Piunikhin has shown that

$$
\phi_{c}^{a b}=\phi_{a b}^{c}= \pm \sqrt{\frac{\langle a, b, c\rangle}{\langle c\rangle}}
$$

(see formula 4.12 of $[\mathbf{P}]){ }^{7,8}$

\footnotetext{
${ }^{7}$ Actually, formula 4.12 of $[\mathbf{P}]$ contains a certain $6-j$-symbol which can however be evaluated.

${ }^{8}$ The meaning of the square root is not specified in $[\mathbf{P}]$.
} 
REMARK. In view of formula $(*)$ above, the equation

$$
\phi_{c}^{a b} \phi_{a b}^{c}=\frac{\langle a, b, c\rangle}{\langle c\rangle}
$$

is equivalent to

$$
K_{a b}^{c} \circ K_{c}^{a b}=\mathrm{id}_{V_{c}}
$$

(assuming $(a, b, c)$ is an admissible triple).

3. Proof of Theorems 1-3. The properties of the $f_{n}$ that we will need are Wenzl's recursion formula (see $\S 1$ ) and the fact that they are augmentation idempotents, which implies the following two equations:

(i)

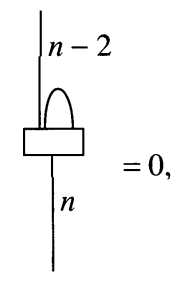

(ii)

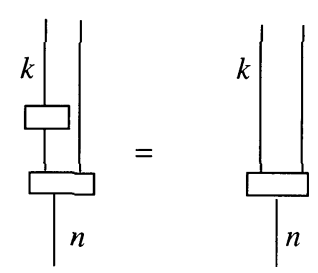

(Recall that writing $n$ beneath a component of a tangle diagram means that this component has to be replaced by $n$ parallel ones.)

LEMMA 1.

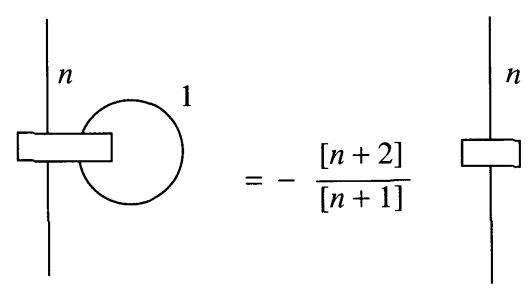

Proof. Let us denote by $g_{n}$ the element of $T_{n}$ represented by the diagram on the L.H.S. Using (ii), one sees that $g_{n}=\lambda f_{n}$ for some constant $\lambda$. The closure $\widehat{g_{n}} \in \mathscr{B}$ of $g_{n}$ satisfies

$$
\left\langle\widehat{g_{n}}\right\rangle_{C}=\langle n+1\rangle=\left\langle e_{n+1}\right\rangle_{C}
$$


(these notations are defined in $\S 2$ ). Hence

$$
\lambda=\frac{\left\langle e_{n+1}\right\rangle_{C}}{\left\langle e_{n}\right\rangle_{C}}=-\frac{[n+2]}{[n+1]}
$$

as asserted.

LEMMA 2. For $j \geq 1$ :
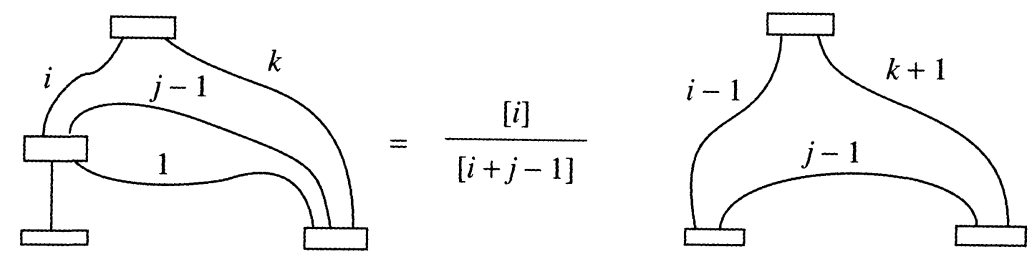

Proof. The proof is by induction on $j$. For $j=1$, this follows from (ii). For $j \geq 2$, we apply Wenzl's formula on the L.H.S. to the box representing $f_{i+j-1}$. The first term of that formula yields zero by (i): hence we get

$$
=\frac{[i+j-2]}{[i+j-1]}
$$

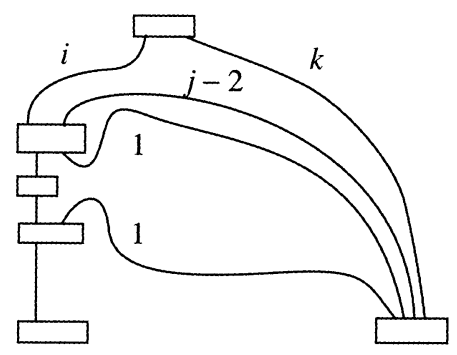

Using (ii), we can draw this as follows:

$$
=\frac{[i+j-2]}{[i+j-1]}
$$

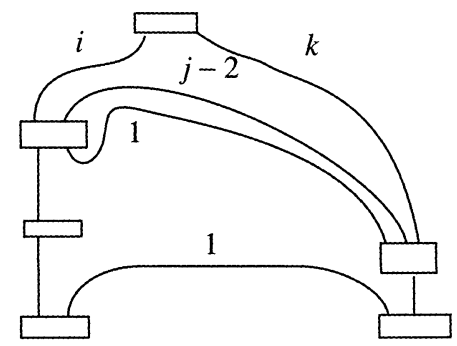

Applying the induction hypothesis to the upper part, we get 


$$
=\frac{[i+j-2]}{[i+j-1]} \frac{[i]}{[i+j-2]}
$$

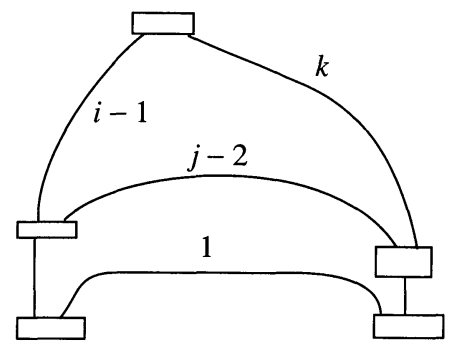

Using (ii) again, the lemma follows.

REMARK. Lemma 2 remains valid for $i=0$ if we make the convention that a diagram is zero if it has a negative number of strings somewhere.

LEMMA 3. $[n+r][m+r]=[n][m]+[n+m+r][r]$.

Proof. This can be verified by a direct calculation. Alternatively, it also follows easily from the equation

$$
e_{i} e_{j}=\sum_{k} e_{k}
$$

(the sum being over all $k$ such that the triple $(i, j, k)$ is admissible) (see $\S 2)$. Details are left to the reader.

Proof of Theorem 1. It is convenient to set

$$
\langle a, b, c\rangle=[i, j, k]
$$

where the $i, j, k$ are the internal colors of a 3 -valent vertex $(a, b, c)$. We have

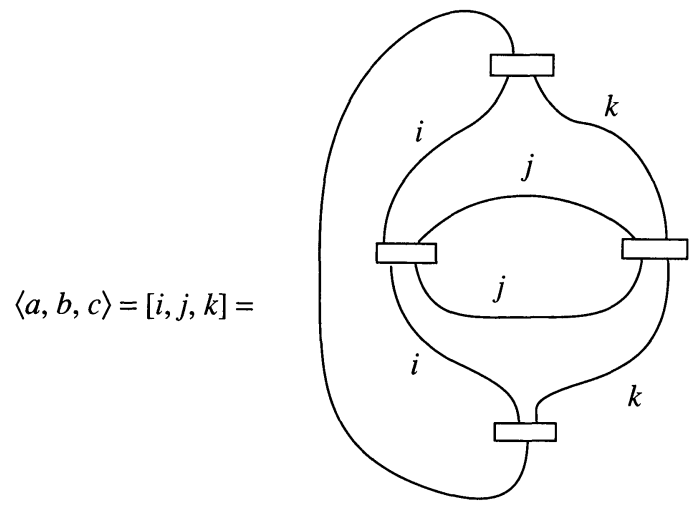


The proof is by induction on $j$. If $j=0$, then clearly

$$
[i, j, k]=\langle i+k\rangle=(-1)^{i+k}[i+k+1]
$$

and the theorem is true in this case. If $j \geq 1$, we apply Wenzl's formula to get
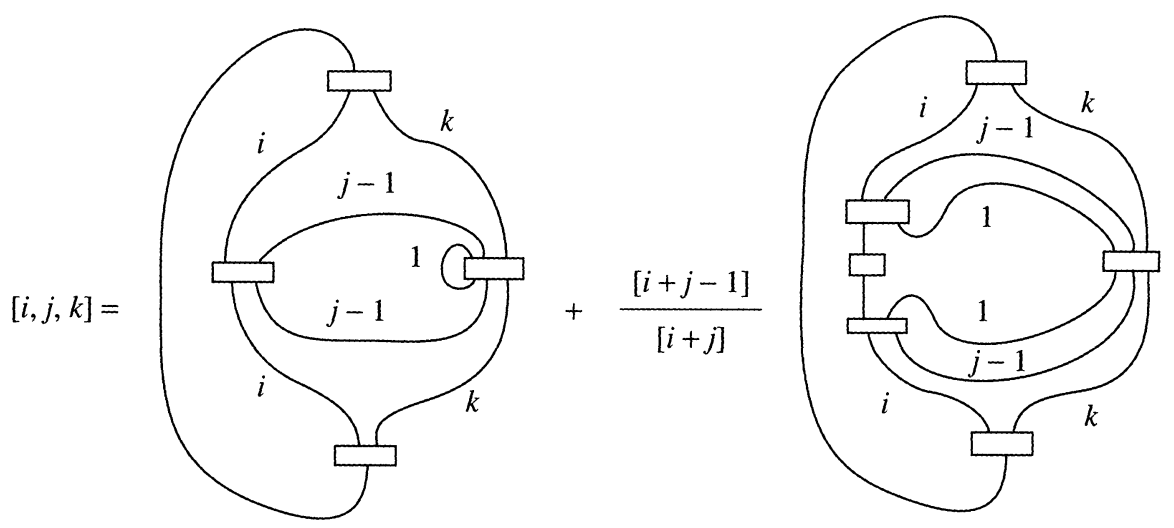

We can simplify this, using Lemmas 1 and 2 . Thus we get the following recursion formula:

$$
\begin{aligned}
{[i, j, k]=} & -\frac{[j+k+1]}{[j+k]}[i, j-1, k] \\
& +\frac{[i]^{2}}{[i+j][i+j-1]}[i-1, j-1, k+1] .
\end{aligned}
$$

Applying the induction hypothesis to the R.H.S., we get after simplifying

$$
\begin{aligned}
& {[i, j, k]} \\
& \quad=(-1)^{i+j+k} \frac{[i+j+k+1] ![i] ![j] ![k] !}{[i+j] ![j+k] ![i+k] !} \frac{[i+j][j+k+1]-[i][k+1]}{[j][i+j+k+1]} .
\end{aligned}
$$

But $[i+j][j+k+1]-[i][k+1]=[j][i+j+k+1]$ because of Lemma 3 , whence the result.

LEMMA 4. For $y \geq 1$
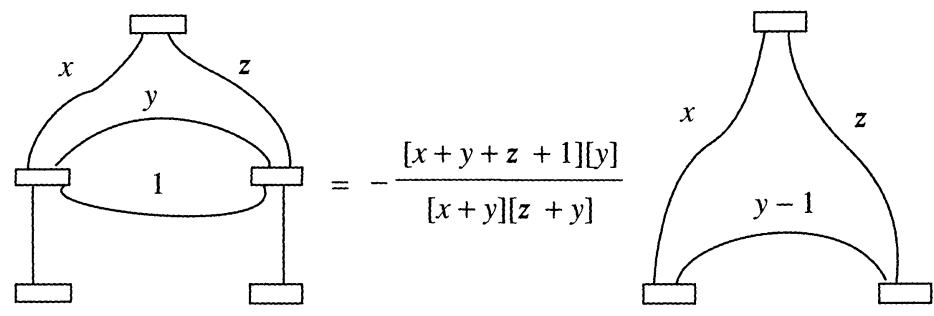
Proof. Wenzl's recursion formula applied to the box representing $f_{x+y}$ yields
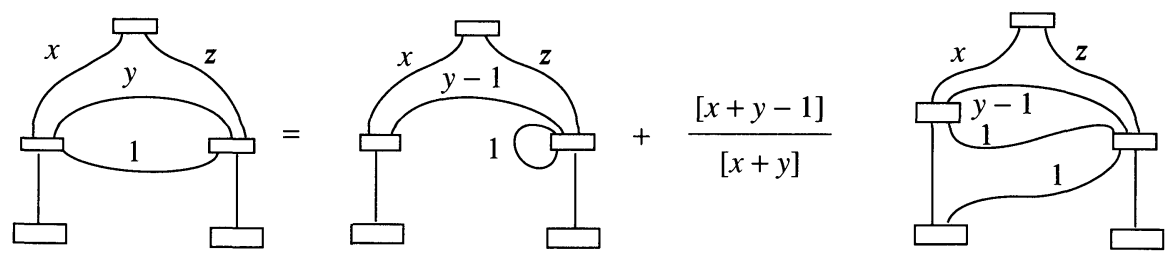

The first diagram on the R.H.S. can be simplified using Lemma 1. The second diagram can be simplified using Lemma 2 twice, as indicated below:

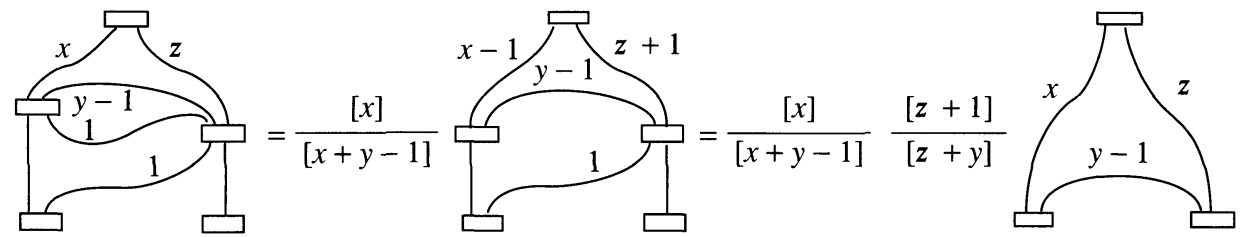

Hence
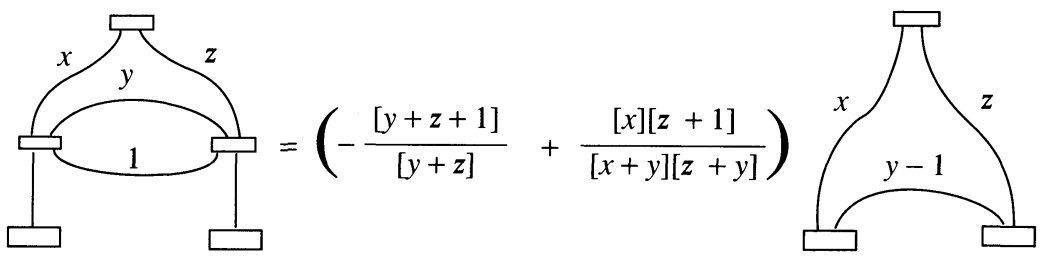

The result now follows from Lemma 3.

Proof of Theorem 2. We start by reformulating Theorem 2 as follows. Notice that the numbers $a_{j}$ and $b_{i}$ defined in Theorem 2 satisfy $\sum a_{j}=\sum b_{i}=\Sigma$ and $\max \left(a_{j}\right) \leq \min \left(b_{i}\right)$. Conversely, it is not hard to see that whenever $a_{1}, a_{2}, a_{3}, a_{4}, b_{1}, b_{2}, b_{3}$ are nonnegative integers satisfying these two conditions, then they uniquely determine $A, B, C$ etc. such that there is a well-defined tetrahedron coefficient. With this in mind, we define

$$
\begin{aligned}
& {\left[\begin{array}{llll}
a_{1} & a_{2} & a_{3} & a_{4} \\
& b_{1} & b_{2} & b_{3}
\end{array}\right]} \\
& \quad=\left\{\begin{array}{ccc}
\frac{[A] ![B] ![C] ![D] ![E] ![F] !}{\prod_{i=1}^{3} \prod_{j=1}^{4}\left[b_{\imath}-a_{j}\right] !}\left\langle\begin{array}{lll}
A & B & E \\
D & C & F
\end{array}\right\rangle & \text { if } \max \left(a_{j}\right) \leq \min \left(b_{i}\right) \\
0 & & \text { if } \max \left(a_{j}\right)>\min \left(b_{i}\right)
\end{array}\right.
\end{aligned}
$$

whenever $\sum a_{j}=\sum b_{i}\left(\right.$ and $\left.a_{j} \geq 0, b_{i} \geq 0\right)$. 
Theorem 2 can now be stated as follows:

$$
\left[\begin{array}{cccc}
a_{1} & a_{2} & a_{3} & a_{4} \\
& b_{1} & b_{2} & b_{3}
\end{array}\right]=\left(\begin{array}{cccc}
a_{1} & a_{2} & a_{3} & a_{4} \\
b_{1} & b_{2} & b_{3}
\end{array}\right)
$$

where the R.H.S. is defined by

$$
\left(\begin{array}{cccc}
a_{1} & a_{2} & a_{3} & a_{4} \\
b_{1} & b_{2} & b_{3}
\end{array}\right)=\sum_{\max \left(a_{j}\right) \leq \zeta \leq \min \left(b_{i}\right)} \frac{(-1)^{\zeta}[\zeta+1] !}{\prod_{i=1}^{3}\left[b_{i}-\zeta\right] ! \prod_{j=1}^{4}\left[\zeta-a_{j}\right] !}
$$

To obtain a recursion formula for $\left[\begin{array}{ccccc}a_{1} & a_{2} & a_{3} & a_{4} \\ & b_{1} & b_{2} & b_{3}\end{array}\right]$, it is convenient to introduce some more notation. Let $i, j, k$ denote the internal colors of the vertex labeled $a_{1}$, and similarly $\alpha, \beta, \gamma$ those of $a_{2}$, and $x, y, z$ those of $a_{3}$, as in the following figure:
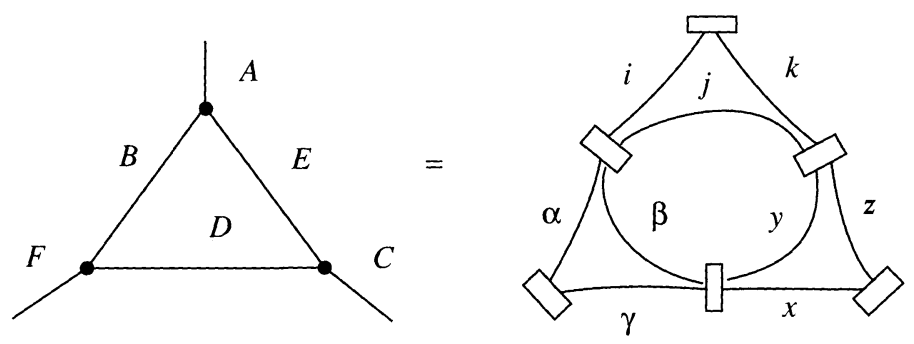

Assume that $j \geq 1$ and $\beta \geq 1$. Applying Wenzl's formula to the edge labeled $B=i+j=\alpha+\beta$, we obtain the above
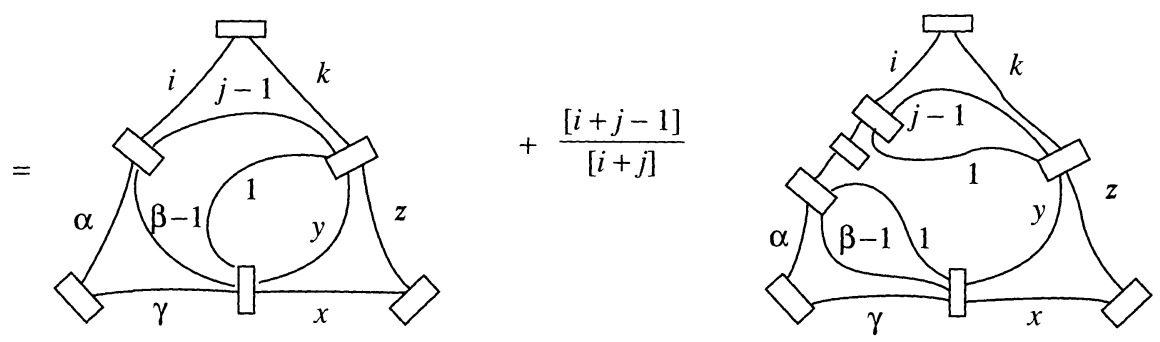

Observe that if $y=0$, then the first term is zero, and if $i=0$ or $\alpha=0$, then the second term is zero (cf. the remark following Lemma 2). We apply Lemmas 4 and 2 and obtain the above 
$=-\frac{[x+y+z+1][y]}{[x+y][y+z]}$
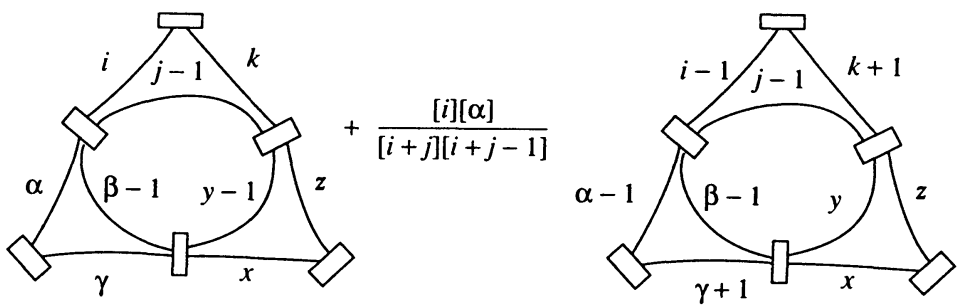

For this recursion formula to be valid it is sufficient to suppose that $j \geq 1$ and $\beta \geq 1$, but we may allow some of the other seven numbers $i, k, \alpha, \gamma, x, y, z$ to be zero. (We make the convention that a diagram is zero if it has a negative number of strings somewhere. This is consistent with the convention that

$$
\left[\begin{array}{cccc}
a_{1} & a_{2} & a_{3} & a_{4} \\
& b_{1} & b_{2} & b_{3}
\end{array}\right]=0
$$

if $\max \left(a_{j}\right)>\min \left(b_{i}\right)$, because the 12 numbers $b_{i}-a_{j}$ are the internal colors of the four vertices of the tetrahedron.) If $j=0$ or $\beta=$ 0 , however, the R.H.S. is zero whereas the L.H.S. may be non-zero. But if we multiply both sides of the recursion formula by $[j][\beta]=$ $\left[b_{1}-a_{4}\right]\left[b_{2}-a_{4}\right]$, the result will be true without restriction. After rewriting things in terms of the $a_{j}$ and the $b_{i}$, we thus obtain:

$$
\begin{aligned}
{\left[b_{1}\right.} & \left.-a_{4}\right]\left[b_{2}-a_{4}\right]\left[\begin{array}{cccc}
a_{1} & a_{2} & a_{3} & a_{4} \\
& b_{1} & b_{2} & b_{3}
\end{array}\right] \\
= & -\left[a_{3}+1\right]\left[a_{1}+a_{2}-b_{3}\right]\left[\begin{array}{cccc}
a_{1}-1 & a_{2}-1 & a_{3}-1 & a_{4} \\
b_{1}-1 & b_{2}-1 & b_{3}-1
\end{array}\right] \\
& +\left[b_{3}-a_{1}+1\right]\left[b_{3}-a_{2}+1\right]\left[\begin{array}{cccc}
a_{1}-1 & a_{2}-1 & a_{3} & a_{4} \\
b_{1}-1 & b_{2}-1 & b_{3}
\end{array}\right] .
\end{aligned}
$$

We now set out to prove

$$
\left[\begin{array}{cccc}
a_{1} & a_{2} & a_{3} & a_{4} \\
& b_{1} & b_{2} & b_{3}
\end{array}\right]=\left(\begin{array}{cccc}
a_{1} & a_{2} & a_{3} & a_{4} \\
b_{1} & b_{2} & b_{3}
\end{array}\right) .
$$

If $\max \left(a_{j}\right)>\min \left(b_{i}\right)$, this is true by definition.

Next, we consider the case where $\max \left(a_{j}\right)=\min \left(b_{i}\right)$. Then (at least) one of the 12 numbers $b_{i}-a_{j}$ is zero, and without loss of generality, we may suppose $y=b_{3}-a_{4}=0$. We proceed by induction on $\min (j, \beta)=\min \left(b_{1}-a_{4}, b_{2}-a_{4}\right)$. If this is zero, then the tetrahedron coefficient reduces to a trihedron coefficient, and the result 
follows easily from Theorem 1 . Now suppose $\min (j, \beta) \geq 1$. The first term on the R.H.S. of our recursion formula being zero, we have

$$
\begin{aligned}
& {\left[\begin{array}{cccc}
a_{1} & a_{2} & a_{3} & a_{4} \\
& b_{1} & b_{2} & b_{3}
\end{array}\right]} \\
& =\frac{\left[b_{3}-a_{1}+1\right]\left[b_{3}-a_{2}+1\right]}{\left[b_{1}-a_{4}\right]\left[b_{2}-a_{4}\right]}\left[\begin{array}{cccc}
a_{1}-1 & a_{2}-1 & a_{3} & a_{4} \\
b_{1}-1 & b_{2}-1 & b_{3}
\end{array}\right] .
\end{aligned}
$$

It is easy to verify that the same relation holds for $\left(\begin{array}{c}\ldots . . \\ \ldots\end{array}\right)$ in place of $\left[\begin{array}{c}\cdots . . \\ \ldots .\end{array}\right]$ (the sum reduces to the term for $\zeta=b_{3}=a_{4}$ ). This yields the induction step. Thus we have shown Theorem 2 in the case $\max \left(a_{j}\right)=$ $\min \left(b_{i}\right)$.

To prove the theorem in the general case, we wish to simplify the recursion formula obtained above. At this point, it is useful to look

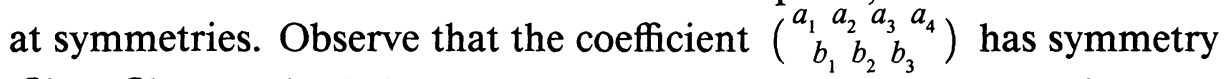
$\mathscr{S}_{4} \times \mathscr{S}_{3}$; that is, it is unchanged under any permutation of the $a_{i}$

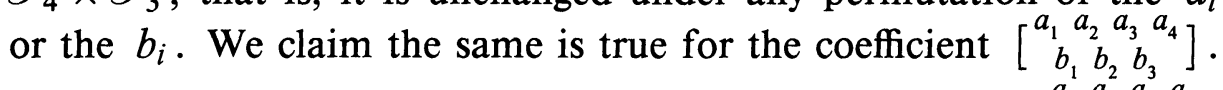
Indeed, it follows from the recursion formula above that $\left[\begin{array}{cccc}a_{1} & a_{2} & a_{3} & a_{4} \\ b_{1} & b_{2} & b_{3}\end{array}\right]$ is unchanged under the permutation $a_{1} \leftrightarrow a_{2}$, and also under the permutation $b_{1} \leftrightarrow b_{2}$. But recall that the recursion formula was obtained after choosing a face of the tetrahedron (the face whose vertices are labeled $\left.a_{1}, a_{2}, a_{3}\right)$ and an edge on the face (the edge joining $a_{1}$ and $a_{2}$ ). It is easy to see that the recursion formulas we can get from making the other choices imply that the coefficient $\left[\begin{array}{cccc}a_{1} & a_{2} & a_{3} & a_{4} \\ b_{1} & b_{2} & b_{3}\end{array}\right]$ indeed has symmetry $\mathscr{S}_{4} \times \mathscr{S}_{3}$.

Hence we can exchange the roles of $a_{2}$ and $a_{3}$ in the recursion formula above. Subtracting the old recursion formula from the new one, we obtain

$$
\begin{aligned}
0= & \left(\left[a_{3}+1\right]\left[a_{1}+a_{2}-b_{3}\right]-\left[a_{2}+1\right]\left[a_{1}+a_{3}-b_{3}\right]\right) \\
& \cdot\left[\begin{array}{cccc}
a_{1}-1 & a_{2}-1 & a_{3}-1 & a_{4} \\
b_{1}-1 & b_{2}-1 & b_{3}-1
\end{array}\right] \\
+ & {\left[b_{3}-a_{1}+1\right]\left[b_{3}-a_{3}+1\right]\left[\begin{array}{cccc}
a_{1}-1 & a_{2} & a_{3}-1 & a_{4} \\
b_{1}-1 & b_{2}-1 & b_{3}
\end{array}\right] } \\
& -\left[b_{3}-a_{1}+1\right]\left[b_{3}-a_{2}+1\right]\left[\begin{array}{cccc}
a_{1}-1 & a_{2}-1 & a_{3} & a_{4} \\
b_{1}-1 & b_{2}-1 & b_{3}
\end{array}\right] .
\end{aligned}
$$

However, applying Lemma 3 with $n=a_{2}-a_{3}, m=a_{1}-b_{3}-1$, 
$r=a_{3}+1$, we find

$$
\begin{gathered}
{\left[a_{3}+1\right]\left[a_{1}+a_{2}-b_{3}\right]-\left[a_{2}+1\right]\left[a_{1}+a_{3}-b_{3}\right]} \\
=-\left[a_{2}-a_{3}\right]\left[a_{1}-b_{3}-1\right] .
\end{gathered}
$$

Hence, the equation above has $\left[b_{3}-a_{1}+1\right]$ (which is equal to $\left.-\left[a_{1}-b_{3}-1\right]\right)$ as a common factor. If $b_{3} \neq a_{1}-1$, we may divide by this common factor, and obtain

$$
\begin{aligned}
& {\left[a_{3}-a_{2}\right]\left[\begin{array}{cccc}
a_{1}-1 & a_{2}-1 & a_{3}-1 & a_{4} \\
b_{1}-1 & b_{2}-1 & b_{3}-1
\end{array}\right] } \\
&=\left[b_{3}-a_{3}+1\right]\left[\begin{array}{cccc}
a_{1}-1 & a_{2} & a_{3}-1 & a_{4} \\
b_{1}-1 & b_{2}-1 & b_{3}
\end{array}\right] \\
&-\left[b_{3}-a_{2}+1\right]\left[\begin{array}{cccc}
a_{1}-1 & a_{2}-1 & a_{3} & a_{4} \\
b_{1}-1 & b_{2}-1 & b_{3}
\end{array}\right] .
\end{aligned}
$$

This is the "simpler" recursion formula we sought. Using Lemma 3 as above, it is not hard to verify that the same formula is true with $\left(\begin{array}{cccc}a_{1} & a_{2} & a_{3} & a_{4} \\ b_{1} & b_{2} & b_{3}\end{array}\right)$ in place of $\left[\begin{array}{cccc}a_{1} & a_{2} & a_{3} & a_{4} \\ b_{1} & b_{2} & b_{3}\end{array}\right]$. We leave this to the reader. (It is true "term by term", that is, even before summing over $\zeta$.) Notice that it follows that our formula remains true if $b_{3}=a_{1}-1$, because in that case we know already that $\left(\begin{array}{l}\cdots . . \\ \ldots\end{array}\right)=\left[\begin{array}{l}\cdots . . \\ \ldots\end{array}\right]$ for the three terms appearing in that formula.

Setting

$$
\left\{\begin{array}{cccc}
a_{1} & a_{2} & a_{3} & a_{4} \\
& b_{1} & b_{2} & b_{3}
\end{array}\right\}=\left[\begin{array}{cccc}
a_{1} & a_{2} & a_{3} & a_{4} \\
b_{1} & b_{2} & b_{3}
\end{array}\right]-\left(\begin{array}{cccc}
a_{1} & a_{2} & a_{3} & a_{4} \\
b_{1} & b_{2} & b_{3}
\end{array}\right)
$$

we thus obtain after a slight change of variables the following final formula:

$$
\begin{aligned}
& {\left[a_{3}-a_{2}+1\right]\left\{\begin{array}{cccc}
a_{1} & a_{2}-1 & a_{3} & a_{4} \\
b_{1} & b_{2} & b_{3}-1
\end{array}\right\}} \\
& =\left[b_{3}-a_{3}\right]\left\{\begin{array}{cccc}
a_{1} & a_{2} & a_{3} & a_{4} \\
b_{1} & b_{2} & b_{3}
\end{array}\right\} \\
& -\left[b_{3}-a_{2}+1\right]\left\{\begin{array}{ccccc}
a_{1} & a_{2}-1 & a_{3}+1 & a_{4} \\
& b_{1} & b_{2} & b_{3}
\end{array}\right\} .
\end{aligned}
$$

We now conclude the proof of Theorem 2 as follows. We must show $\left\{\begin{array}{cccc}a_{1} & a_{2} & a_{3} & a_{4} \\ b_{1} & b_{2} & b_{3}\end{array}\right\}=0$. We use double induction on $\Sigma=\sum a_{j}=\sum b_{i}$ and on $d=\min \left(b_{i}\right)-\max \left(a_{j}\right)$. For $\Sigma=0$ there is nothing to show, and 
if $d=0$, then we know already the result. If $\Sigma \geq 1$ and $d \geq 1$, we look at our final formula. By the hypothesis of the induction on $\Sigma$, the L.H.S. is zero. Since $\left\{\begin{array}{c}a_{1} a_{2} a_{3} a_{4} \\ b_{1} b_{2} b_{3}\end{array}\right\}$ is unchanged under any permutation of the $a_{j}$, we may assume that $\max \left(a_{j}\right)=a_{3}$; hence by the hypothesis of the induction on $d$, the second term of the R.H.S. is zero. Hence

$$
\left[b_{3}-a_{3}\right]\left\{\begin{array}{cccc}
a_{1} & a_{2} & a_{3} & a_{4} \\
b_{1} & b_{2} & b_{3}
\end{array}\right\}=0 .
$$

But we assumed $d \geq 1$; hence $\left[b_{3}-a_{3}\right]$ is non-zero, and the result follows.

This completes the proof of Theorem 2 .

Proof of Theorem 3. We reproduce here an argument of O. Viro [V]. One has the following two equations:
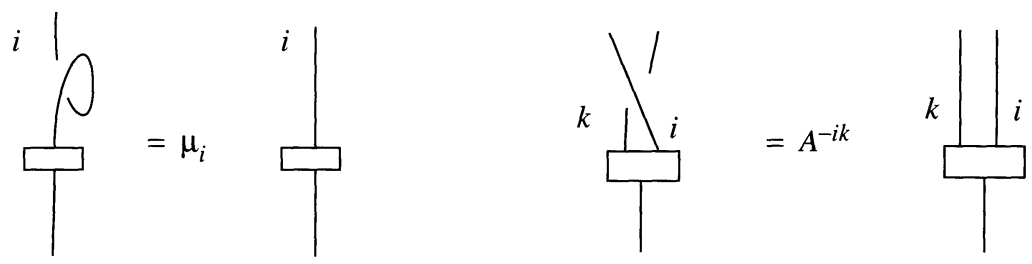

where $\mu_{i}=(-1)^{i} A^{i^{2}+2 i}$. Indeed, the first equation is an immediate consequence of the equation $t e_{i}=\mu_{i} e_{i}$ of [BHMV1] (see also [Li1, Li2]), and the second equation follows from applying the Kauffman relations to the $i k$ crossings of the diagram, together with the fact that the box represents the augmentation idempotent $f_{k+i}$. Hence
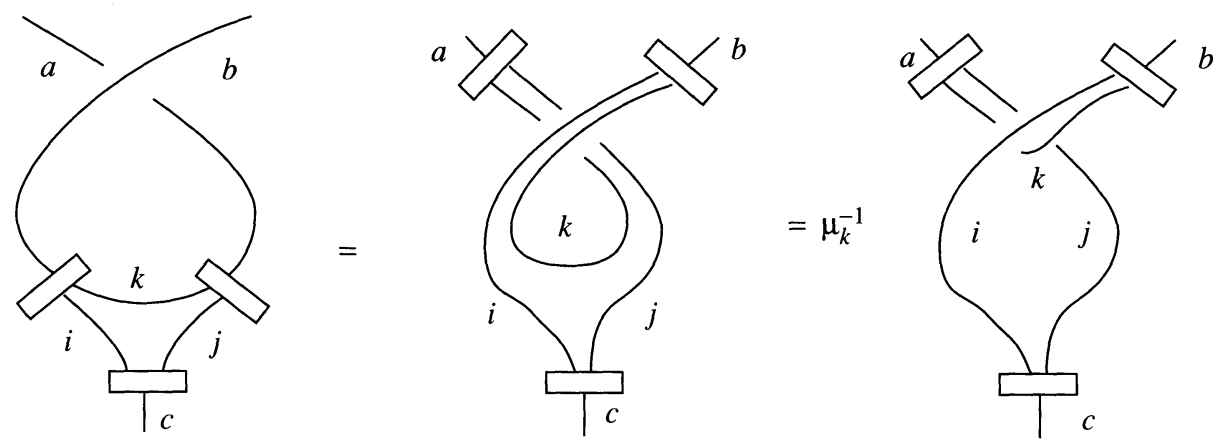


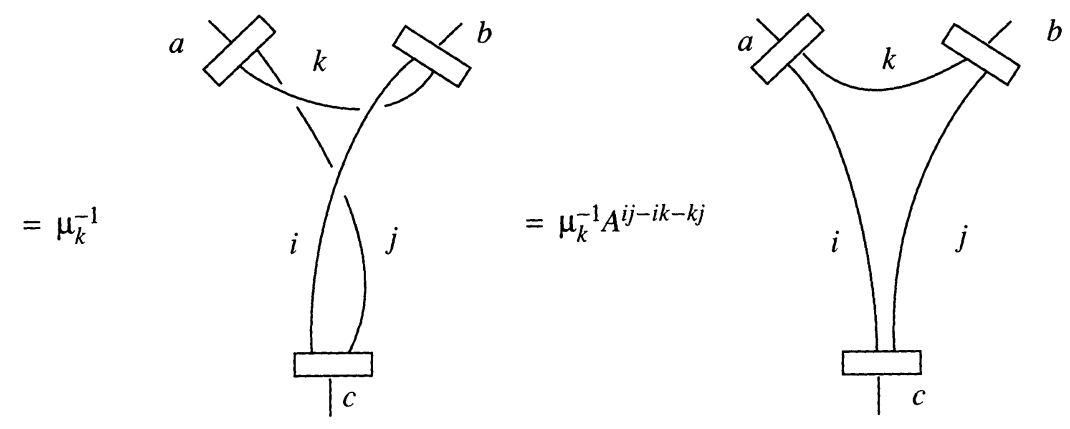

whence

$$
\delta(c ; a, b)=\mu_{k}^{-1} A^{i j-i k-k j}=(-1)^{k} A^{-k^{2}-2 k+i j-i k-k j}
$$

as asserted.

REMARK. One has

$$
\delta(c ; a, b)^{2}=\frac{\mu_{c}}{\mu_{a} \mu_{b}} .
$$

This follows immediately from Theorem 3 .

\section{REFERENCES}

[BHMV1] C. Blanchet, N. Habegger, G. Masbaum and P. Vogel, Three-manifold invariants derived from the Kauffman bracket, Topology, 31, No. 4, (1992), 685-699.

[BHMV2] _ Remarks on the Three-manifold Invariants $\theta_{p}$, NATO Advanced Research Workshop and Conference on Operator Algebras, Mathematical Physics, and Low-Dimensional Topology, Istanbul, July 1991 (to appear).

[BHMV3] _ Topological quantum field theories derived from the Kauffman bracket, preprint May 1992 (in revised from January 1993).

[BL] L. C. Biedenharn and J. D. Louck, Angular momentum in quantum physics, Encyclopedia of Mathematics and its Applications, vol. 8, AddisonWesley 1981.

[J1] V. F. R. Jones, Index of subfactors, Invent. Math., 72 (1983), 1-25.

[J2] A polynomial invariant for links via von Neumann algebras, Bull. Amer. Math. Soc., 12 (1985), 103-111.

[Ka1] L. H. Kauffman, State models and the Jones polynomial, Topology, 26 (1987), 395-401.

[Ka2] Knots, spin networks, and 3-manifold invariants, (preprint 1990).

[KM] R. C. Kirby and P. Melvin, The 3-manifold invariants of Witten and Reshetikhin-Turaev for sl(2, C), Invent. Math., 105 (1991), 473-545.

[KR] A. N. Kirillov and N. Yu. Reshetikhin, Representations of the algebra $U_{q}\left(\mathbf{s l}_{2}\right)$, q-orthogonal polynomials, and invariants of links, LOMI preprint 1988. 
[Li1] W. B. R. Lickorish, Three-manifold invariants and the Temperly-Lieb algebra, Math. Ann., 290 (1991), 657-670.

[Li2] Calculations with the Temperley-Lieb algebra, Comment. Math. Helv., 68 (1992), 571-591.

[MS] H. R. Morton and P. Strickland, Jones polynomial invariants for knots and satellites, Math. Proc. Cambridge Philos. Soc., 109 (1991), 83-103.

[P] S. Piunikhin, Turaev-Viro and Kauffman invariants for 3-manifolds coincide, J. Knot Theory and its Ramifications, vol. I no. 2, (1992), 105-135.

[RT] N. Yu. Reshetikhin and V. G. Turaev, Invariants of 3-manifolds via link polynomials and quantum groups, Invent. Math., 103 (1991), 547-597.

[TV] V. G. Turaev and O. Ya. Viro, State sum invariants of 3-manifolds and quantum 6-j-symbols, Topology, 31 (1992), 865-902.

[V] O. Ya. Viro, private communication.

[We] H. Wenzl, On sequences of projections, C. R. Math. Rep. Acad. Sci. Canada, IX (1987), 5-9.

Received March 15, 1992.

UNIVERSITÉ DE NANTES

URA $n^{0} 758$ DU CNRS

2 RUE DE LA HOUSSINIËRE

44072 Nantes Cedex 03, France

AND

UNIVERSITE PARIS VII

URA $n^{0} 212$ DU CNRS

Case postale 7012, 2 Place Jussieu

75251 Paris Cedex 05, France 



\section{CONTENTS}

K. Auinger, Free products of combinatorial strict inverse semigroups . . . . . . . . 201

T. Dana-Picard, Generic 8-dimensional algebras with mixed basis-graph . . . . . . 229

D. Dimovski, One-parameter fixed point indices . . . . . . . . . . . . . . . . . 263

K. S. Druschel, Oriented orbifold cobordism . . . . . . . . . . . . . . . . . . . 299

A. D. Helfer, Conjugate points on spacelike geodesics or pseudo-self-adjoint MorseSturm-Liouville systems . . . . . . . . . . . . . . . . . . . . . 321

J. G. Heywood and O. D. Walsh, A counter-example concerning the pressure in the Navier-Stokes equations, as $t \rightarrow 0^{+} \ldots \ldots \ldots \ldots \ldots \ldots \ldots \ldots \ldots, \ldots \ldots \ldots$

G. Masbaum and P. Vogel, 3-valent graphs and the Kauffman bracket . . . . . . 361

R. Stong, The Jones polynomial of parallels and applications to crossing number 383

Vol. 164, No. 2 


\section{PACIFIC JOURNAL OF MATHEMATICS}

Volume $164 \quad$ No. $2 \quad$ June 1994

Free products of combinatorial strict inverse semigroups

201

KARL AUINGER

Generic 8-dimensional algebras with mixed basis-graph

229

THIERRY DANA-PICARD

One-parameter fixed point indices

263

DONCO DIMOVSKI

Oriented orbifold cobordism

299

KIMBERLY SUE DRUSCHEL

Conjugate points on spacelike geodesics or pseudo-self-adjoint

Morse-Sturm-Liouville systems

ADAM D. HELFER

A counterexample concerning the pressure in the Navier-Stokes

351 equations, as $t \rightarrow 0^{+}$

JOHN GROVES HeYwOOD and OWEN WALSH

3-valent graphs and the Kauffman bracket

Gregor Masbaum and PierRe Vogel

The Jones polynomial of parallels and applications to crossing number 383

RICHARD ANDREW STONG 